\title{
Stereotactic radiation treatment for benign meningiomas
}

\author{
Andrew E. H. Elia, M.D., Ph.D., Helen A. Shih, M.D., and Jay S. Loeffler, M.D. \\ Department of Radiation Oncology, Massachusetts General Hospital, Boston, Massachusetts
}

\begin{abstract}
$\checkmark$ Meningiomas are the second most common primary tumor of the brain. Gross-total resection remains the preferred treatment if achievable with minimal morbidity. For incompletely resected or inoperable benign meningiomas, 3D conformal external-beam radiation therapy can provide durable local tumor control in 90 to $95 \%$ of cases. Stereotactic radiosurgery (SRS) and fractionated stereotactic radiotherapy (SRT) are highly conformal techniques, using steep dose gradients and stereotactic patient immobilization. Stereotactic radiosurgery has been used as an alternative or adjuvant therapy to surgery for meningiomas in locations, such as the skull base, where operative manipulation may be particularly difficult. Stereotactic radiotherapy is useful for larger meningiomas $(>3-3.5 \mathrm{~cm})$ and those closely approximating critical structures, such as the optic chiasm and brainstem. Although SRS has longer follow-up than SRT, both techniques have excellent 5-year tumor control rates of greater than $90 \%$ for benign meningiomas. Stereotactic radiotherapy has toxicity equivalent to that of radiosurgery, despite its biased use for larger meningiomas with more complicated volumes. Reported rates of imaging-documented regression are higher for radiosurgery, but neurological recovery is relatively good with both techniques. Stereotactic radiosurgery and fractionated SRT are complementary techniques appropriate for different clinical scenarios. (DOI: 10.3171/FOC-07/10/E5)
\end{abstract}

KEY WoRDS • meningioma • stereotactic radiosurgery • stereotactic radiotherapy

$\mathrm{M}$ ENINGIOMAS account for 15 to $25 \%$ of all primary brain tumors and have a reported annual incidence between one and 10 per $100,000 .{ }^{47}$ Surgery is the preferred treatment for benign meningiomas whenever complete resection can be achieved with reasonable morbidity, resulting in 5-, 10-, and 15-year PFS rates of 93, 80, and $68 \%$, respectively. ${ }^{31}$ However, complete resection is not possible in 20 to $30 \%$ of presenting patients. ${ }^{31,42}$ In these cases, subtotal resection has inferior results, with 5-, 10-, and 15-year PFS rates of 63, 45, and 9\%, respectively. ${ }^{31}$

Studies from the 1980s showed that EBRT could provide durable local tumor control in incompletely resected benign meningiomas. As shown in Table 1, its addition to subtotal resection increased 5- to 8-year PFS rates from 40 to $48 \%$ to 68 to $88 \%$ in three series with a median followup of 6 to 8 years. ${ }^{4,30,44}$ Long-term local control rates were 70 to $85 \%$, regardless of whether meningiomas were irradiated in the adjuvant setting or as initial primary therapy (Table 2). ${ }^{12,14}$ After the advent of CT planning, Goldsmith and coworkers ${ }^{15}$ showed that local control using radiation therapy could rival that of gross-total resection. They de-

Abbreviations used in this paper: $\mathrm{CT}=$ computed tomography; EBRT = external-beam radiation therapy; GKS = Gamma Knife surgery; GTV = gross tumor volume; ICA = internal carotid artery; LINAC $=$ linear accelerator; $\mathrm{MR}=$ magnetic resonance; ONSM = optic nerve sheath meningioma; PFS = progression-free survival; SRS = stereotactic radiosurgery; SRT $=$ stereotactic radiotherapy. monstrated that 5-year PFS in the post-1980 era with the aid of more modern techniques was $98 \%$ compared with $77 \%$ in patients treated before $1980(\mathrm{p}=0.002)$. More conformal therapies allow a higher radiation dose, and these authors demonstrated improved 10-year PFS rates (93\%) for doses greater than 52 Gy than for doses 52 Gy or less $(65 \%, p=0.04)$. These high control rates have been replicated in more modern series, such as that conducted between 1984 and 2001 by Mendenhall et al., ${ }^{28}$ in which the 5-, 10-, and 15-year local control rates were 95, 92, and $92 \%$, respectively.

In addition to 3D treatment planning, improved conformality has come through the use of SRS and fractionated SRT. Stereotactic radiosurgery is useful for treating meningiomas at locations where surgical intervention might threaten neurovascular integrity, such as the cavernous sinus or posterior parasagittal region. Fractionated SRT, on the other hand, is helpful in cases in which SRS has limitations, such as with larger tumors or those that arise near critical structures, like the optic chiasm or brainstem. Stereotactic radiotherapy is particularly promising for ONSMs, where few other alternatives are appropriate. The purpose of this review is to describe these techniques, present outcome data from retrospective experiences, and compare indications for these modalities. We will focus specifically on World Health Organization Grade I benign meningiomas. 


\section{Stereotactic Radiosurgery}

\section{Technique of SRS}

Stereotactic radiosurgery is a technique of external radiation that classically uses multiple convergent beams to deliver a high single dose of radiation to a small volume. Its hallmark is a steep dose gradient at the target boundary, which allows sparing of adjacent normal tissue. This rapid dose falloff is produced by the intersection of multiple beams from different directions and a high degree of collimation. The term stereotaxis refers to knowing the exact location of an intracranial target, with respect to an external 3D coordinate system, which is referenced to the rotational isocenter of a radiation treatment unit and thus is used to direct treatment. Stereotactic radiosurgery today is performed using three basic modalities: LINAC, Gamma Knife, and protons.

Linear accelerator-based radiosurgery uses X-ray beams that are produced by the collision of accelerated electrons with a metal target. Multiple noncoplanar arcs converge at a single isocenter, where a nearly spherically shaped dose distribution is created. These arcs are produced by gantry rotation during radiation, and each is defined by a different couch angle. By adjusting the number, lengths, angles, and weights of individual arcs, one can reduce irradiation of adjacent critical structures. In addition to noncoplanar arcs, a single dynamic arc can be used, and it is produced by simultaneous rotation of the gantry and couch. Most LINAC radiosurgery units use tertiary circular collimators that further reduce beam divergence and thereby protect normal tissues. For higher conformality that departs from a spherical shape, multiple isocenters are possible, however, at the cost of greater inhomogeneity and longer treatment times. Thus most LINAC radiosurgery today is performed with a single isocenter. To increase conformality with a single isocenter, miniature multileaf tertiary collimators have been used, achieving dose distributions that are both highly conformal and highly homogeneous.

The Gamma Knife uses $201{ }^{60} \mathrm{Co}$ sources that release two photons (gamma rays) with an average energy of 1.2 $\mathrm{MeV}$ for each spontaneous decay. The sources are arranged on the surface of a hemispherically shaped shell, each aimed at a single isocenter $40 \mathrm{~cm}$ from each source. The beams produced by these sources are shaped by secondary collimators on a helmet, with diameters of $4,8,14$, or 18 $\mathrm{mm}$. Plugging of these collimators with solid billets allows the deletion of individual beams. At installation, Gamma Knife units have an initial dose rate of $4 \mathrm{~Gy} /$ minute, and with a half-life of 5.28 years, the primary sources must be replaced every 7 to 10 years. Because of the low energy of the isotope source, most treatments use a normalization of $50 \%$. The total time to irradiate a single isocenter with the Gamma Knife is typically a few minutes, making the use of multiple isocenters practical. With multiple isocenters commonly used, Gamma Knife plans therefore tend to be more conformal but less homogeneous than LINAC-based plans. Recent modifications to the Gamma Knife unit have improved the treatment time for multiisocenter plans by including a semiautomatic positioning system that moves the patient with computer-driven motors.

With steep dose gradients in radiosurgery, accurate alignment of the plan's isocenter with the physical isocenter of the treatment unit is critical. Both LINAC and Gamma
Knife systems use invasive stereotactic head rings, which are attached to the patient using screws that rest on the outer table of the skull. An imaging reference frame is then attached to the head ring during CT scanning or MR imaging, and coordinates identifying the isocenter are specified with respect to this frame. Using the reference frame, the patient is then aligned with the beam delivery unit before treatment. In recent years, image guidance systems have been developed which allow for precise stereotaxy without the need for an external coordinate system. Such image guidance systems include auxiliary orthogonal x-ray imaging in real time (the CyberKnife), megavoltage fan beam helical CT scanning (TomoTherapy), and kilovoltage cone beam CT scanning.

Tumors appropriate for radiosurgery include those that are generally smaller than 3 to $3.5 \mathrm{~cm}$, with little or no surrounding edema, and located in sites where dose constraints for adjacent critical structures (such as the optic apparatus and brainstem) can be maintained. Benign meningiomas are potentially ideal targets for radiosurgery, as they are usually well-circumscribed, noninvasive, and easily recognized on neuroimaging due to homogeneous contrast enhancement and the presence of a dural tail, which is characteristic but not pathognomic. The types of meningiomas frequently targeted by SRS are skull base and parasagittal, because microsurgery in these areas can be associated with a high risk of cranial nerve, brainstem, and vascular damage.

For treatment planning in both radiosurgery and fractionated radiation therapy, target definition is generally superior with MR imaging because it offers better tumor enhancement and no bone artifacts. However, CT-based planning provides direct measurement of tissue photon attenuation, which is necessary for precise dose calculation. Furthermore, the spatial data provided by CT and MR imaging do not always overlap. Khoo and coworkers ${ }^{19}$ showed that MR imaging-defined meningioma volumes were generally greater in size but not necessarily inclusive of CT-defined volumes. We thus prefer MR imaging/CT scanning fusion for smaller meningiomas, as it combines the complementary spatial information of CT scanning and MR imaging. For larger meningiomas, CT-based planning by itself is usually sufficient. Computed tomography is performed with cuts of $3 \mathrm{~mm}$ or thinner after contrast enhancement, whereas MR imaging is performed with 1- to 3-mm cuts using Gd-enhanced T1-weighted images, obtained with a magnet no less than 1.5 tesla. For benign meningiomas, the clinical target volume is essentially equivalent to the GTV and does not include areas of edema. Postoperatively, the clinical target volume may be expanded beyond the residual radiographic GTV to include microscopic disease based on intraoperative findings or appreciation of the preoperative dural base. After irradiation of benign meningiomas, surveillance MR images should be obtained at 6 and 12 months, and then annually thereafter. If the patient is believed to be at a low risk for recurrence (that is, after near gross-total resection), imaging can be spaced to every 2 years after 5 years have passed.

\section{Outcome of SRS}

Since the early 1990s, numerous reports have appeared in the literature on the use of radiosurgery for menin- 


\section{TABLE 1}

Improved local control by EBRT after subtotal resection of meningiomas*

\begin{tabular}{lcccc}
\hline \hline & & & \multicolumn{2}{c}{ PFS (\%) $\dagger$} \\
\cline { 4 - 5 } \multicolumn{1}{c}{ Authors \& Year } & Yrs of Study & FU (yrs) & STR & STR \& RT \\
\hline Barbaro et al., 1987 & $1968-1978$ & 6.5 & 40 & 68 \\
Taylor et al., 1988 & $1964-1985$ & 5.0 & 43 & 85 \\
Miralbell et al., 1992 & $1968-1986$ & 8.0 & 48 & 88 \\
\hline
\end{tabular}

Abbreviations: FU = follow-up; RT = radiotherapy; STR = subtotal resection.

$\dagger$ Barbaro et al., provide crude local control data. Taylor, Miralbell, and colleagues' data are actuarial.

giomas. Table 3 lists published series since 2001 with more than 100 patients per study and a minimum follow-up of 3 years. These retrospective series have demonstrated 5-year local control rates that range between 86 and $99 \%$, tumor regression rates of 28 to $70 \%$, symptom improvement in 8 to $65 \%$ of patients, and toxicity of 2.5 to $13 \%$. We will highlight some of the most recent and largest studies with the longest follow-up and then glean a few lessons from these series about treatment plan design. In virtually all of these studies, radiosurgery was used as both initial primary therapy and as adjuvant treatment after surgery.

Between 1990 and 1998 Stafford and colleagues ${ }^{43}$ treated 168 benign intracranial meningiomas with either primary $(41 \%)$ or adjuvant $(59 \%)$ radiosurgery. Patients were treated to a median marginal dose of $16 \mathrm{~Gy}$, and 5-year PFS after a median follow-up of 40 months was $93 \% .{ }^{43}$ Subsequently, Pollock et al. ${ }^{36}$ compared outcomes from a subset of these patients to surgical outcomes. They reviewed 188 benign meningiomas that were treated with either resection (126 tumors) or GKS alone (62 tumors) to a mean marginal dose of 17.7 Gy. After a median follow-up of 64 months, the 7-year PFS for radiosurgery was not significantly different from that for a Simpson Grade 1 resection (95\% compared with $96 \%, \mathrm{p}=0.94$ ), which represents gross removal of all tumor and involved dura and bone. Furthermore, radiosurgery had superior tumor control than less complete excisions, including Simpson Grade 2 (82\% PFS at 7 years, $p<0.05)$ and Grade 3 to 4 (34\% PFS at 7 years, $\mathrm{p}<0.001)$. As expected, the ability to achieve a Grade 1 resection strongly correlated with location, occurring in more than $95 \%$ of convexity meningiomas but less than $33 \%$ of skull base, falx, and tentorial lesions. The authors concluded that radiosurgery should be strongly considered as the primary treatment modality for tumors involving these difficult locations. ${ }^{36}$

The cavernous sinus is one skull base location where meningiomas are particularly difficult to resect, because of a high risk of vascular and cranial nerve damage. Nicolato et al. ${ }^{34}$ reviewed their experience with 122 benign cavernous sinus meningiomas treated with primary (60 tumors) or adjuvant (62 tumors) GKS to a mean marginal dose of 14.6 Gy. With a median follow-up of 48.9 months, the PFS at 5 years was $96.5 \%$. Also remarkably, neurological function improved in $65 \%$ and remained stable in $32 \%$ of patients. Of interest, neurological recovery was greater in patients treated with primary rather than adjuvant SRS after surgery (79\% compared with $61 \%, \mathrm{p}<0.05$ ), probably because surgical insult caused a certain amount of irreparable damage to cranial nerve function. On imaging, tumors regressed in $62 \%$ of cases and remained stable in $36 \%$. The authors noted that longer follow-up resulted in higher rates of tumor regression, with shrinkage seen in $80 \%$ of patients followed for longer than 30 months and only $44 \%$ of patients followed for less than 30 months $(\mathrm{p}<0.0002)$.

Lee and coworkers ${ }^{26}$ also reported their experience with cavernous sinus meningiomas. They treated 159 tumors between 1987 and 2000 with either primary (83 tumors) or adjuvant (76 tumors) GKS to a median marginal dose of 13 Gy. After a median follow-up of 3 years, the 5-year PFS was $93 \%$ for the entire population. For patients who underwent primary radiosurgery, the 5-year PFS was 96.9\%, whereas it was only $79.6 \%$ for those who had adjuvant SRS after surgery. There should be no biological difference between tumors subjected to previous resection and those treated primarily with radiosurgery. The authors suggested that the disparate results may arise from the fact that resected tumors are more difficult to define on imaging, due to postoperative enhancement. They therefore recommended waiting several weeks postresection before performing adjuvant SRS.

The radiosurgery group at the Medical University of Graz in Austria has demonstrated excellent long-term results in their treatment of meningiomas, most recently updated by Kreil et al. ${ }^{23}$ Between 1992 and 1999, they treated 200 patients, 101 with primary radiosurgery and 99 with adjuvant radiosurgery. The median marginal dose was 12 Gy, median target volume $6.5 \mathrm{~cm}^{3}$, median isodose line $45 \%$, and median number of isocenters six. Patients were followed for a median of 7.9 years, and the 5- and 10-year PFS rates were excellent at 98.5 and $97.2 \%$, respectively. Preexisting neurological symptoms (such as visual field deficits, diplopia, trigeminal neuralgia, exophthalmos, and vertigo) improved in $41.5 \%$ of patients, remained stable in $54 \%$, and deteriorated in only $4.5 \%$. Radiological tumor regression occurred in 57\% and tumor stability in $42 \%$. Complications occurred in five patients $(2.5 \%)$, four of whom had transient deficits of increased seizure activity, headache aggravation, and new trigeminal neuralgia, and one with permanent visual deterioration.

Kollová and coworkers ${ }^{20}$ have published the most recent large ( $>100$ patients) study on radiosurgery for meningiomas. They described their experience between 1992 and 1999 in treating 325 benign intracranial meningiomas with a median tumor volume of $4.4 \mathrm{~cm}^{3}$, using either primary $(70 \%)$ or adjuvant $(30 \%)$ GKS. Patients were treated with a median marginal dose of 12.6 Gy by using a median of six isocenters. After a median follow-up of 60 months, the 5 -year local control was excellent at $97.9 \%$. Improvement in neurological symptoms (such as imbalance, oculomotor palsy, trigeminal neuralgia, seizure, hemiparesis, and vertigo) occurred in $61.9 \%$. Imaging-documented tumor regression occurred in $69.7 \%$ of patients, and stability was noted in $27.8 \%$. Permanent toxicity occurred in $5.7 \%$ of patients and included seizures, trigeminal symptoms, hemiparesis, oculomotor palsy, vertigo, cognitive changes, and hearing loss.

What have retrospective studies taught us about optimal radiosurgery dose for meningiomas? In early reports, such as that by Engenhart et al. ${ }^{10}$ in 1990, the authors used high doses between 10 and 50 Gy (median 29 Gy) that resulted 
A. E. H. Elia, H. A. Shih, and J. S. Loeffler

in late severe symptomatic edema in $29 \%$ of patients. Later series involved more moderate doses and had much less toxicity. In 1998, Kondziolka et al. ${ }^{21}$ demonstrated symptomatic edema of $16 \%$ with a median marginal dose of $15 \mathrm{~Gy}$ over a range of 9 to $32 \mathrm{~Gy}$. On multivariate analysis, they showed that local control was no better for doses of at least $15 \mathrm{~Gy}$ than for doses less than $15 \mathrm{~Gy}$. In recent years, median doses have decreased further with no apparent detriment in outcome. Thus, the studies of Kreil et al.,., Kollová et al. ${ }^{20}$ and Lee et al. ${ }^{26}$ demonstrate 5-year PFS rates of 98.5, 97.9 , and $93 \%$, respectively, with median doses of $12,12.6$, and 13 Gy, respectively (Table 3). In the study by Kollová et al., doses less than 12 Gy were associated with an increase in tumor volume after radiation $(p=0.047)$, whereas doses greater than 16 Gy were associated with increased edema $(\mathrm{p}<0.001)$ but with no benefit on local control. Thus, it appears that doses between 12 and 16 Gy are sufficient to provide excellent tumor control with acceptable toxicity. In their recent review, Chin et al. ${ }^{7}$ recommended 12 to $14 \mathrm{~Gy}$ for tumors greater than $3 \mathrm{~cm}, 16 \mathrm{~Gy}$ for tumors 1 to $3 \mathrm{~cm}$, and $18 \mathrm{~Gy}$ for tumors less than $1 \mathrm{~cm}$. In addition to tumor volume, other factors that should influence dose include prior radiation treatment and proximity to sensitive structures.

Inclusion of the dural tail in the target volume is a point of controversy in meningioma treatment planning. Given that dural tails are distal to the bulk of meningioma lesions, their targeting can result in excessive normal tissue radiation. DiBiase et al. ${ }^{9}$ examined this issue in their review of 139 meningiomas, which had a median tumor volume of $4.5 \mathrm{~cm}^{3}$ and were treated with either primary ( 85 tumors) or adjuvant (52 tumors) GKS to a median dose of 14 Gy. For the entire population, the 5-year PFS was $86 \%$ over a median follow-up of 4.5 years. On univariate analysis, inclusion of the dural tail resulted in better local control $(92 \%$ versus $78 \%, \mathrm{p}<0.035$ ). However, concerns about this analysis have included a lack of significance on multivariate analysis, no information on patterns of tumor failure, and correlation of dural tail inclusion with decreased conformality $(\mathrm{p}=0.04) .{ }^{38}$ Because microscopic invasion of the dural tail is typically confined to a distance of 1 to $2 \mathrm{~mm}$ beyond the gross tumor ${ }^{33}$ we believe that inclusion of the first several millimeters of adjacent dura is likely to be sufficient.

Researchers at some institutions have attempted partial tumor irradiation, which has led to poor tumor control rates. Shin et al. ${ }^{41}$ reviewed their experience with 40 cavernous sinus meningiomas treated with GKS. On univariate analysis, they found that partial tumor irradiation was associated with local recurrence $(\mathrm{p}<0.0001)$. When tumors were completely covered with a minimal marginal dose of 14 Gy (22 patients), local control was $100 \%$ over a median follow-up of 37 months. However, when portions of tumors were treated with only 10 to 12 Gy (15 patients) or no radiation at all (three patients), local control was 80 and $0 \%$, respectively. Malik et al. ${ }^{27}$ treated 309 intracranial meningiomas with a median tumor volume of $7.3 \mathrm{~cm}^{3}$ between 1994 and 2000 with either primary (136 tumors) or adjuvant (173 tumors) radiosurgery. ${ }^{27}$ The median margin dose was $20 \mathrm{~Gy}$, and the average number of isocenters was 6.5 . In $52 \%$ of cases, however, part of the tumor received less than the prescription dose to protect neurological function, most commonly at the optic apparatus in cavernous sinus meningiomas. Although this approach led to a
TABLE 2

Primary or adjuvant therapy with EBRT for meningiomas

\begin{tabular}{lccc}
\hline \hline \multicolumn{1}{c}{ Authors \& Year } & $\begin{array}{c}\text { Yrs of } \\
\text { Study }\end{array}$ & $\begin{array}{c}\text { Median } \\
\text { FU (yrs) }\end{array}$ & $\begin{array}{c}5-\mathrm{yr} \\
\text { PFS }(\%)\end{array}$ \\
\hline Forbes \& Goldberg, 1984 & $1970-1982$ & 3.8 & $72 \dagger$ \\
Glaholm et al., 1990 & $1963-1983$ & 6.7 & 73 \\
Goldsmith et al., 1994 & $1967-1980$ & 3.3 & 77 \\
& $1981-1990$ & 3.3 & 98 \\
Mendenhall et al., 2003 & $1984-2001$ & 5.1 & 98 \\
\hline
\end{tabular}

$* \mathrm{FU}=$ follow-up.

$\dagger$ In this study the PFS duration was 4 years.

low complication rate of $3 \%$, it gave a 5-year local control of only $87 \%$ for benign tumors, which is lower than that for most other large modern series (Table 3).

\section{Toxicity of SRS}

As shown in Table 3, a recent trend toward lower radiation doses has resulted in lower toxicity rates. Thus, median marginal doses of 12 Gy reported by Kreil et al. ${ }^{23}$ and 14.6 Gy reported by Nicolato et al. ${ }^{34}$ resulted in low complication rates of 2.5 and $4.1 \%$, respectively. Flickinger et al. ${ }^{11}$ have documented a decrease in toxicity over time in their study of 219 meningiomas diagnosed by imaging criteria alone. They showed that the risk of postradiosurgery sequelae was higher in patients treated before rather than after 1991 (22.9\% versus 5.3\%, p 0.01), during which time radiation doses were lower and stereotactic MR imaging was used rather than CT. The median marginal dose was 17 Gy (range 10-20 Gy) between 1987 and 1991, and it was 14 Gy (range 8.9-20 Gy) between 1991 and 2000.

The toxicity from radiosurgery of meningiomas comes mostly from symptomatic edema or damage to cranial nerves located on the skull base. Tishler et al ${ }^{45}$ examined the tolerance of the second through sixth cranial nerves to radiosurgery and found a significantly increased incidence of complications for patients receiving greater than $8 \mathrm{~Gy}$ to any part of the optic apparatus compared with those who received less than 8 Gy (24\% compared with $0 \%, p=$ 0.009). ${ }^{45}$ Later studies showed that doses up to 10 Gy could be delivered to the optic apparatus without problems. ${ }^{25}$ The oculomotor nerves of the cavernous sinus can easily tolerate doses greater than $20 \mathrm{~Gy},{ }^{25,45}$ whereas the trigeminal nerve in Meckel's cave is more sensitive, and its maximum tolerated dose has generally been considered $19 \mathrm{~Gy} .{ }^{32}$ For the seventh and eighth cranial nerves, lowering the dose to $12 \mathrm{~Gy}$ in the treatment of vestibular schwannomas has reduced the incidence of facial nerve injury to less than $5 \% .35$

Vascular occlusion after radiosurgery is rare but has been documented in the treatment of cavernous sinus meningiomas, with an incidence of 1 to $2 \%$, as recently reviewed. ${ }^{3}$ In these cases, it has occurred in a delayed fashion, usually 14 to 60 months after SRS. Stafford et al. ${ }^{43}$ reported ICA occlusion in two of 66 patients with cavernous sinus meningiomas 35 and 60 months after GKS. The radiation dose exceeded 25 Gy in both cases. Roche et al. ${ }^{37}$ described one of 92 patients with a cavernous sinus meningioma who developed ICA stenosis 14 months after GKS, and her calculated ICA dose was 36 Gy. Radiosurgery experiments in mammalian models suggest that occlusion develops as a 
TABLE 3

Stereotactic radiosurgery for meningiomas published since $2001 *$

\begin{tabular}{|c|c|c|c|c|c|c|c|c|c|}
\hline Authors \& Year & $\begin{array}{l}\text { Years } \\
\text { of Study }\end{array}$ & $\begin{array}{c}\text { No. of } \\
\text { Patients }\end{array}$ & $\begin{array}{l}\text { Median } \\
\text { FU (mos) }\end{array}$ & $\begin{array}{c}\text { Median } \\
\text { Dose (Gy) }\end{array}$ & $\begin{array}{c}5-Y r \\
\text { PFS (\%) }\end{array}$ & $\begin{array}{c}\text { Clinical } \\
\operatorname{RR}(\%) \dagger\end{array}$ & $\begin{array}{c}\text { Tumor } \\
\text { Regression } \\
(\%) \neq\end{array}$ & $\begin{array}{c}\text { Median } \\
\text { TV }\left(\mathrm{cm}^{3}\right)\end{array}$ & $\begin{array}{c}\text { Compli- } \\
\text { cation } \\
\text { Rate }(\%)\end{array}$ \\
\hline Stafford et al., 2001 & 1990-1998 & 168 & 40 & 16 & 93 & 8 & 56 & - & 13 \\
\hline Lee et al., 2002 & 1987-2000 & 159 & 35 & 13 & 93 & 29 & 34 & 6.5 & 6.9 \\
\hline Nicolato et al., 2002 & 1993-2002 & 122 & 48.9 & 14.6 & 96.5 & 65 & 61 & 8.1 & 4.1 \\
\hline Pollock et al., 2003 & 1990-1997 & 62 & 64 & 17.7 (mean) & 95 (at 7 yrs) & 13 & - & - & 10 \\
\hline DiBiase et al., 2004 & 1992-2000 & 162 & 54 & 14 & 86 & - & 28 & 4.5 & 8.3 \\
\hline Kreil et al., 2005 & 1992-1999 & 200 & 94.8 & 12 & 98.5 & 41.5 & 57 & 6.5 & 2.5 \\
\hline Malik et al., 2005 & 1994-2000 & 309 & 44 & 20 & 87 & - & - & 7.3 & 3 \\
\hline Kollová et al., 2007 & 1992-1999 & 325 & 60 & 12.6 & 97.9 & 61.9 & 69.7 & 4.4 & 5.7 \\
\hline
\end{tabular}

$* \mathrm{RR}=$ response rate; $\mathrm{TV}=$ tumor volume; $-=$ not stated.

$\uparrow$ Clinical response rate refers to improvement in preexisting neurological deficits.

† Tumor regression is precisely defined in Stafford et al. as decrease in tumor size greater than $2 \mathrm{~mm}$. In other studies, it is specified less definitively as any decrease in tumor volume.

result of luminal narrowing associated with endothelial damage. ${ }^{3}$

\section{Fractionated SRT}

\section{Fractionated SRT Technique}

Fractionated SRT combines the precision of stereotactic positioning and the steep dose gradients of arc-based radiation with the radiobiological effect of fractionation. The advantage of fractionation is that it helps to spare normal tissue by allowing time for the repair of sublethal damage between radiation fractions. In essence, therefore, SRT differs from SRS simply based on the number of fractions. However, treatment units for SRT have generally been limited to LINACs, for which relocatable frames have been developed. To attain the same level of stereotactic precision as radiosurgery, accurate head immobilization is required. However, invasive frames would not be practical because repeated frame applications are necessary over multiple weeks. Relocatable immobilization systems have therefore been designed and include bite block devices, such as the Gill-Thomas-Cosman frame for adults ${ }^{13}$ and the TarbellLoeffler-Cosman frame for children, ${ }^{22}$ or reinforced thermoplastic mask-based systems. ${ }^{39}$

\section{Fractionated SRT Outcome}

Studies of fractionated SRT are less common and have shorter follow-up than those for radiosurgery (Table 4). The Royal Marsden group initially published their early experience in $1999^{1}$ and recently updated it in 2002..$^{18}$ Between 1994 and 1999, they treated 41 benign skull base meningiomas with primary (15 tumors) or adjuvant (26 tumors) SRT. All patients were immobilized in a Gill-Thomas-Cosman relocatable frame, and treatment was delivered on an LINAC using customized blocks or a multileaf collimator to doses of 50 to $55 \mathrm{~Gy}$ in 30 to 33 daily fractions. Tumors were relatively large with a median GTV of $17.9 \mathrm{~cm}^{3}$. With a short median follow-up of 21 months, the actuarial local control at 3 years was $100 \%$. Tumor regression occurred in $22 \%$ and was stable in $66 \%$, and clinical improvement (vision or cranial nerve function) occurred in $27 \%$.
Selch et al. ${ }^{40}$ conducted another small study, involving 45 benign cavernous sinus meningiomas, treated with primary (16 tumors) or adjuvant (29 tumors) SRT between 1997 and 2002. The median tumor volume was $14.5 \mathrm{~cm}^{3}$, and the optic apparatus in $66 \%$ of cases was compressed. Patients were immobilized with a thermoplastic face mask and treated with a Novalis LINAC to a median dose of 50.4 Gy using a single isocenter and multileaf collimator, giving a median isodose of $90 \%$. With a median follow-up of 36 months, the 3-year PFS rate was $97.4 \%$. Tumor regression occurred in $18 \%$ of patients and tumor stability in $80 \%$. Preexisting neurological complaints improved in $20 \%$ of patients and were stable in the remaining patients. The authors indicated that field shaping with a micromultileaf collimator gave conformal (conformality index $=2.2$ ) and homogeneous (homogeneity index $=1.11$ ) radiation without the use of multiple isocenters. ${ }^{40}$

The University of Heidelberg group has published the largest series of SRT for meningiomas. Originally published in 2001 with only a 3-year follow-up, ${ }^{8}$ they recently updated their experience with 317 benign or atypical skull base meningiomas treated with either primary (97 tumors) or adjuvant (220 tumors) SRT between 1985 and 2001.29 Tumors were quite large with a median target volume of $33.6 \mathrm{~cm}^{3}$. Patients were immobilized with a thermoplastic mask and treated with LINAC-based SRT to a median dose of 57.6 Gy. With a median follow-up of 5.7 years, the recurrence-free survival was $98 \%$ at 3 years, $90 \%$ at 5 years, and $89 \%$ at 10 years. Tumor regression occurred in $23 \%$ of patients and stability in $70 \%$. Preexisting neurological deficits improved in $43 \%$ of patients, whereas worsening occurred in $8.2 \%$. Analysis of prognostic factors showed that volumes greater than $60 \mathrm{~cm}^{3}(\mathrm{p}<0.001)$ and doses of 55 Gy or less $(p<0.09)$ had worse tumor control, whereas extent of surgery did not matter, confirming earlier findings in reports on EBRT for treating meningiomas.

The most recently published series of SRT for meningiomas is that of Henzel et al. Their experience at two hospitals in Germany involved 224 skull base meningiomas treated between 1997 and 2003 with SRT (183 tumors), hypofractionated SRT (30 tumors), and SRS (11 tumors). Stereotactic radiotherapy was performed on a LINAC with 
A. E. H. Elia, H. A. Shih, and J. S. Loeffler

TABLE 4

Fractionated SRT for intracranial meningiomas

\begin{tabular}{|c|c|c|c|c|c|c|c|c|c|}
\hline Authors \& Year & $\begin{array}{c}\text { Years } \\
\text { of Study }\end{array}$ & $\begin{array}{c}\text { No. } \\
\text { of } \\
\text { Patients }\end{array}$ & $\begin{array}{c}\text { Median } \\
\text { FU } \\
\text { (mos) }\end{array}$ & $\begin{array}{c}\text { Median } \\
\text { Dose } \\
\text { (Gy) }\end{array}$ & PFS $(\%)$ & $\begin{array}{c}\text { Clinical } \\
\text { RR } \\
(\%)\end{array}$ & $\begin{array}{c}\text { Tumor } \\
\text { Regres- } \\
\text { sion }(\%)^{*}\end{array}$ & $\begin{array}{c}\text { Median } \\
\text { TV } \\
\left(\mathrm{cm}^{3}\right)\end{array}$ & $\begin{array}{c}\text { Compli- } \\
\text { cation } \\
\text { Rate }(\%)\end{array}$ \\
\hline Alheit et al., 1999 & 1994-1999 & 41 & 21 & $50-55$ & 100 (at $3 \mathrm{yrs}$ ) & 27 & 22 & 17.9 & 9.8 \\
\hline Selch et al., 2004 & 1997-2002 & 45 & 36 & 50.4 & 97.4 (at 3 yrs) & 20 & 18 & 14.5 & 2 \\
\hline Milker-Zabel et al., 2005 & 1985-2001 & 317 & 68 & 57.6 & 90.0 (at 5 yrs) & 43 & 23 & 33.6 & 2.5 \\
\hline Henzel et al., $2006^{17}$ & $1997-2003$ & 224 & 36 & 55.8 & 96.9 (at 5 yrs) & 43 & 45 & 9.1 & 2.5 \\
\hline
\end{tabular}

* Tumor regression is precisely defined in Henzel et al. ${ }^{17}$ as decrease in tumor diameter greater than $2 \mathrm{~mm}$. In other studies, it is specified less definitively as any decrease in tumor volume.

1- to 3-mm multileaf collimators and always one isocenter to a median dose of 55.8 Gy. Hypofractionated SRT involved either $40 \mathrm{~Gy}$ in 10 fractions, 25 Gy in 5 fractions, or $35 \mathrm{~Gy}$ in 7 fractions, and SRS doses ranged between 15 and 18 Gy. With a median follow-up of 36 months, the 5year PFS rate was $96.9 \%$ for the entire population. Unfortunately, the three treatment modalities were not analyzed separately. The imaging response rate was $45 \%$, as defined by tumor shrinkage greater than $2 \mathrm{~mm}$ using quantitative imaging, and clinical response was $43 \% .{ }^{17}$

Optic nerve sheath meningiomas constitute one subset of tumors for which SRT plays a unique role. Classically, these tumors have presented with the pathognomic triad of painless visual loss, optic atrophy, and optociliary shunt vessels. In reality, however, these three symptoms rarely occur simultaneously, and other manifestations include propotosis, afferent pupillary defects, and visual field detects. On imaging, they appear as diffuse tubular enlargement of the optic nerve, resulting in "tram-tracking" on axial views and concentric "doughnut" thickening on coronal views. Traditionally, neuroophthalmologists have advised that these lesions be observed until vision is lost, at which point resection can occur. Upfront surgery is not performed because the tumors have an intimate circumferential relationship to both the optic nerve and central retinal artery. An attempt at resection causes blindness in nearly all cases, through inevitable damage to the pial vascular supply. Stereotactic radiosurgery is not possible for ONSMs given that such treatment would entail irradiating directly through the optic nerve. This leaves fractionated radiation as virtually the last resort.

Small study size and short follow-up have limited analyses of ONSMs. However, three studies have been published involving at least 20 patients, and all have shown that SRT provides excellent symptom improvement with minimal toxicity. Between 1996 and 2001, Andrews and colleagues $^{2}$ treated 30 optic nerve sheath meningiomas with SRT to a median dose of 54 Gy. After a median follow-up of 22 months, improvement in visual acuity and/or fields occurred in $42 \%$, stability in 50\%, and deterioration in only $9 \%$. Complications occurred in $13 \%$ (four of 30 patients) and included vision loss in two, optic neuritis in one, and transient orbital pain in one. Between 1994 and 2000 , Becker and coworkers ${ }^{6}$ treated 42 eyes (16 with primary and 26 with secondary ONSMs arising intracranially and extending into the optic canal) with SRT to a median dose of 54 Gy. After a median follow-up of 35.5 months, visual acuity improved in 19\% (eight of 42 eyes) and was stable in $81 \%$ ( 34 of 42 eyes). Of 38 visual fields examined, improvement was seen in 31.6\% (12 of 38) and stability in $65.8 \%$ (25 of 38). Complications included endocrin dysfunction in four patients $(9.5 \%)$, whose tumors extended to the pituitary gland, but no visual toxicity was observed. Last, Baumert et al. ${ }^{5}$ reported on 22 symptomatic ONSMs treated at four European institutions between 1996 and 2003 with SRT to a median dose of 54 Gy. After a median follow-up of 20 months, vision improved in 73\% (16 of 22 ), remained stable in $23 \%$ (five of 22), and worsened in $4.5 \%$ (one of 22). Complications occurred in only one patient $(4.3 \%)$, who had radiation retinitis and vitreous hemorrhage.

Given the excellent functional outcome in these studies, which we have also observed in our own experience (J.S.L.) in treating more than 30 optic nerve sheath meningiomas, we believe fractionated SRT is the treatment of choice for this group of tumors. Although rates of imagingdocumented tumor regression are low (Table 5), visual improvement occurs in 19 to $73 \%$ of patients. We believe SRT should be recommended over observation, even in cases of incidentally diagnosed ONSMs, as visual restoration is virtually impossible after blindness occurs. Surgery should be reserved only for cases of rapid visual loss or after blindness has already come to pass. Tumor control has been $100 \%$ in all studies (Table 5), but definite conclusions regarding efficacy and toxicity can only be drawn after longer follow-up.

\section{Fractionated SRT Toxicity}

Common acute toxicities of fractionated SRT are generally mild and include fatigue, skin erythema, and patchy alopecia. Occasionally, intracranial swelling can result in headache, nausea, or exacerbation of previous neurological deficits. In a study by Selch et al. ${ }^{40}$ transient acute morbidities occurred in 18\% of patients (eight of 45) and included headache in four, fatigue in three, and retroorbital pain in one. In a larger study by Henzel et al., ${ }^{17}$ transient low-grade (Grade 1-2) acute toxicity occurred in $43.5 \%$ of patients, whereas clinically significant ( $\geq$ Grade 3 ) acute toxicity was present in only $2.5 \%$.

Late toxicity occurs less frequently in studies of SRT and has ranged between 2 and 13\% (Tables 4 and 5). In the study by Selch et al., ${ }^{40}$ no cranial neuropathy, endocrine dysfunction, or cognitive decline was observed, but an ipsilateral stroke 6 months after SRT did occur in a 57-year-old patient, for whom no other causative factor could be iden- 
TABLE 5

Fractionated SRT for ONSMs

\begin{tabular}{|c|c|c|c|c|c|c|c|c|c|}
\hline Authors \& Year & $\begin{array}{l}\text { Yrs of } \\
\text { Study }\end{array}$ & $\begin{array}{c}\text { No. } \\
\text { of } \\
\text { Patients }\end{array}$ & $\begin{array}{l}\text { Median } \\
\text { FU (mos) }\end{array}$ & $\begin{array}{l}\text { Median } \\
\text { Dose } \\
\text { (Gy) }\end{array}$ & $\begin{array}{c}\% \text { Crude } \\
\text { Local } \\
\text { Control }\end{array}$ & $\begin{array}{c}\text { Visual } \\
\text { Improve- } \\
\text { ment }(\%)^{*}\end{array}$ & $\begin{array}{c}\text { Tumor } \\
\text { Regres- } \\
\text { sion }(\%) \dagger\end{array}$ & $\begin{array}{c}\text { Median } \\
\text { TV } \\
\left(\mathrm{cm}^{3}\right)\end{array}$ & $\begin{array}{l}\text { Compli- } \\
\text { cation } \\
\text { Rate }(\%)\end{array}$ \\
\hline Andrews et al., 2002 & 1996-2001 & 30 & 22 & 54.0 & 100 & 42 & 13.0 & 2.9 & 13 \\
\hline Becker et al., 2002 & 1994-2000 & 42 & 35.5 & 54.0 & 100 & $\begin{array}{l}19 \text { (acuity) } \\
31.6 \text { (fields) }\end{array}$ & 2.6 & - & 9.5 \\
\hline Baumert et al., 2004 & 1996-2003 & 22 & 20 & 54.0 & 100 & 73 & 4.8 & 1.7 & 4.3 \\
\hline
\end{tabular}

* Visual improvement involves either enhanced visual acuity or enhanced visual fields.

$\dagger$ Tumor regression is not uniformly defined in these studies but specified generally as any reduction in tumor volume.

tified, giving a toxicity rate of $2 \%$ (one of 45 ). In the study by Jalali et al., ${ }^{18}$ late complications occurred in four patients $(9.8 \%)$ and included hypopituitarism in two, visual deterioration in one, and cognitive impairment in one. In the larger study by Milker-Zabel et al., ${ }^{29}$ clinically significant $(\geq$ Grade 3) late toxicity was observed in eight patients (2.5\%) and included visual deterioration in two, trigeminal neuralgia in one, and tinnitus in five. As described earlier, toxicity rates for ONSM have ranged between 4.3 and $13 \%$, with visual loss (due to optic neuritis or retinitis) occurring in 0 to $10 \%$ of patients.

\section{Radiosurgery Compared With Fractionated SRT}

Both SRS and fractionated SRT use stereotactic positioning and sharp dose gradients to attain high conformality. Fractionation in SRT provides the additional radiobiological benefit of normal tissue sparing by allowing for repair between consecutive treatments. Most modern series have involved doses of 12 to 16 Gy for radiosurgery and 50 to $54 \mathrm{~Gy}$ in 1.8 - to $2.0-\mathrm{Gy}$ daily fractions for SRT. Using the linear-quadratic formalism for biological effectiveness, these dose ranges should have equivalent radiobiological effect, if one assumes an $\alpha / \beta$ ratio of 2 for meningiomas as a late responding tissue. ${ }^{24}$ Of note, some have pointed out that because meningiomas and brain are both late-responding tissues with similar $\alpha / \beta$ ratios, the theoretical benefit of fractionation with respect to toxicity should be minimal. Thus, what do published studies actually reveal regarding the relative outcomes of SRS and fractionated SRT?

One apparent difference between SRS and fractionated SRT is the radiological response rate (Tables 3-5). For radiosurgery, tumor regression on serial imaging occurs in 28 to $69.7 \%$ of patients, whereas this frequency is only 2.6 to $45 \%$ for fractionated SRT. This may imply an increased radiobiological effect of delivering radiation in a large, single fraction. However, one confounding issue is that SRT series tend to have shorter follow-up periods, and as we have discussed previously, radiological regression is known to increase with longer follow-up. Another concern is the lack of uniformity among studies as to the definition of tumor regression, defined precisely in some series as greater than 2-mm shrinkage observed on MR imaging ${ }^{16,17}$ but specified more ambiguously in other studies as any measurable reduction in tumor volume. Lastly, greater tumor regression with radiosurgery may be related to the fact that tumor volumes tend to be smaller in cases of SRS, and more shrinkage typically occurs in tumors with smaller volumes. Henzel and coworkers ${ }^{16}$ prospectively treated 84 benign skull base meningiomas with SRT and showed tumor volume smaller than $11.1 \mathrm{~cm}^{3}$ predicted for better radiological response rate on multivariate analysis $(27.6 \%$ for volumes $<11.1 \mathrm{~cm}^{3}$ and $21.7 \%$ for volumes $\geq 11.1$ $\mathrm{cm}^{3}, \mathrm{p}$ 0.02). Using quantitative techniques, these authors demonstrated that mean tumor volume after SRT shrank by $16.6,24.5,33.2$, and $36 \%$ at $6,12,24$, and 36 months, respectively, approaching a steady state that has yet to be defined. ${ }^{16}$

Although radiological response may be higher for radiosurgery than fractionated SRT, clinical response does not appear to differ, with values ranging between 8 and $65 \%$ for SRS and 19 and $73 \%$ for fractionated SRT. A lack of correlation between tumor shrinkage and clinical response may seem nonintuitive but has been borne out in retrospective studies. The aforementioned quantitative volume analysis of Henzel et al. ${ }^{16}$ showed no association between these factors $(p=0.41)$. A disconcordance between radiological and clinical response is especially evident in optic nerve sheath meningiomas, in which tumor regression rates are 4.8 to $13 \%$ but symptomatic improvement occurs in up to $73 \%$ of patients (Table 4 ).

In addition to clinical response, neither local tumor control nor toxicity differ significantly between SRS and fractionated SRT. Radiosurgery series have demonstrated 86 to 99\% local control at a median follow-up of 35 to 94.8 months, whereas fractionated SRT series have a local control rate of 90 to $100 \%$ at a median follow-up of 20 to 68 months. Follow-up for SRT studies is generally shorter than that for radiosurgery series, but there is no reason to suspect that, ultimately, long-term control for fractionated SRT will be any less than that for fractionated EBRT. Toxicity for SRS ranges between 2.5 and $13 \%$ and between 0 and $9.8 \%$ for fractionated SRT.

A randomized controlled trial between radiosurgery and fractionated SRT is unlikely ever to be performed. However, one group did compare their single-institution experience with the two modalities. ${ }^{46}$ Between 1991 and 2002, these authors treated 135 benign intracranial meningiomas with radiosurgery (63 tumors) or fractionated SRT (72 tumors) to mean doses of 15.7 and 48.4 Gy, respectively. Results were virtually identical with crude local control rates of 92\% (SRS) and 97\% (SRT), imaging-documented response rates of 35\% (SRS) and 33\% (SRT), clinical response rates of 35\% (SRS) and 32\% (SRT), and complication rates of $5 \%$ in both modalities. The authors cau- 
tioned that the slightly higher local control rate for fractionated SRT was probably secondary to a shorter median follow-up of 24 months, relative to a median follow-up of 40 months for SRS.

These virtually identical outcomes beg the question why a patient would choose 6 weeks of fractionated radiotherapy when the same local control with identical toxicity might be attained with a single day of radiosurgery. The achievement of SRT is exactly this: identical toxicity, despite larger median tumor volumes (Tables 4 and 5) in more critical locations. Multiple series have demonstrated that larger tumor volume is associated with higher rates of both recurrence and toxicity in radiosurgery. On multivariate analysis, Kondziolka and coworkers ${ }^{21}$ showed that tumor volume of at least $7.5 \mathrm{~cm}^{3}$ was predictive of local recurrence $(77 \%$ compared with $56 \%, \mathrm{p}=0.002)$, whereas DiBiase and colleagues ${ }^{9}$ demonstrated that volume of at least $10 \mathrm{~cm}^{3}$ was associated with local failure $(92 \% \mathrm{com}-$ pared with $68 \%, \mathrm{p}=0.038$ ). As for toxicity rates, Flickinger and coworkers ${ }^{11}$ showed that postradiosurgery sequelae tended to increase with treatment volume $(\mathrm{p}=$ 0.053), whereas Kollová and colleagues ${ }^{20}$ demonstrated that tumor volume greater than $10 \mathrm{~cm}^{3}$ predicted for increased edema ( $p<0.001$ on multivariate analysis) and permanent neurological deficits $(p=0.002$ on multivariate analysis). ${ }^{20}$ Thus, the advantage of SRT is that tumor control and complication rates similar to that of SRS can be attained, despite larger and more complicated volumes.

On this basis, neither SRS nor SRT is an inherently superior technique, but rather each is appropriate for different clinical scenarios. Both should be available to complement each other, providing safer treatment options for patients. Stereotactic radiosurgery is beneficial for skull base and parasagittal locations, where the likelihood of gross-total resection is limited. We recommend using SRT over SRS when the tumor is large ( $>3$ to $3.5 \mathrm{~cm}$ ), approaches within 2-4 $\mathrm{mm}$ of critical structures (such as the optic apparatus or brainstem), and in all cases of optic nerve sheath meningioma.

\section{Conclusions}

Gross-total resection is the preferred treatment for benign meningiomas that can be resected with reasonable morbidity. It is particularly desirable for patients requiring immediate decompression for symptomatic relief. Radiation therapy is appropriate for both incompletely resected meningiomas and as a primary treatment option for inoperable tumors. Stereotactic radiosurgery is a convenient single-day alternative to surgery for meningiomas located in areas such as the skull base or parasagittal region, where attempted resection would put critical neurovascular structures at risk for damage. Stereotactic radiotherapy uses the same stereotactic positioning and sharp dose gradients as radiosurgery to attain high conformality but has the additional radiobiological advantage of fractionation to allow normal tissue sparing. It is therefore useful for larger tumors and those that abut critical structures. Both SRS and SRT for incompletely resected or inoperable benign meningiomas have excellent tumor control rates. Complications are also low for both techniques if SRS is restricted to small lesions that are not abutting critical structures. Whereas imaging-documented regression is slow, neurological recovery does occur in some patients. The amount of timing of such regression is interesting from a radiobiological perspective, but it is not important for the patient's outcome.

\section{References}

1. Alheit H, Saran FH, Warrington AP, Rosenberg I, Perks J, Jalali $\mathrm{R}$, et al: Stereotactically guided conformal radiotherapy for meningiomas. Radiother Oncol 50:145-150, 1999

2. Andrews DW, Faroozan R, Yang BP, Hudes RS, Werner-Wasik M, Sergott RC, et al: Fractionated stereotactic radiotherapy for the treatment of optic nerve sheath meningiomas: preliminary observations of 33 optic nerves in 30 patients with historical comparison to observation with or without prior surgery. Neurosurgery 51:890-904, 2002

3. Barami K, Grow A, Brem S, Dagnew E, Sloan AE: Vascular complications after radiosurgery for meningiomas. Neurosurg Focus 22(3):E9, 2007

4. Barbaro NM, Gutin PH, Wilson CB, Sheline GE, Boldrey EB, Wara WM: Radiation therapy in the treatment of partially resected meningiomas. Neurosurgery 20:525-528, 1987

5. Baumert BG, Villa S, Studer G, Mirimanoff RO, Davis JB, Landau $\mathrm{K}$, et al: Early improvements in vision after fractionated stereotactic radiotherapy for primary optic nerve sheath meningioma. Radiother Oncol 72:169-174, 2004

6. Becker G, Jeremic B, Pitz S, Buchgeister M, Wilhelm H, Schiefer $\mathrm{U}$, et al: Stereotactic fractionated radiotherapy in patients with optic nerve sheath meningioma. Int J Radiat Oncol Biol Phys 54: 1422-1429, 2002

7. Chin LS, Szerlip NJ, Regine WF: Stereotactic radiosurgery for meningiomas. Neurosurg Focus 14(5):E6, 2003

8. Debus J, Wuendrich M, Pirzkall A, Hoess A, Schlegel W, Zuna I, et al: High efficacy of fractionated stereotactic radiotherapy of large base-of-skull meningiomas: long-term results. J Clin Oncol 19:3547-3553, 2001

9. DiBiase SJ, Kwok Y, Yovino S, Arena C, Naqvi S, Temple R, et al: Factors predicting local tumor control after gamma knife stereotactic radiosurgery for benign intracranial meningiomas. Int J Radiat Oncol Biol Phys 60:1515-1519, 2004

10. Engenhart R, Kimmig BN, Hover KH, Wowra B, Sturm V, van Kaick G, et al: Stereotactic single high dose radiation therapy of benign intracranial meningiomas. Int J Radiat Oncol Biol Phys 19:1021-1026, 1990

11. Flickinger JC, Kondziolka D, Maitz AH, Lunsford LD: Gamma knife radiosurgery of imaging-diagnosed intracranial meningioma. Int J Radiat Oncol Biol Phys 56:801-806, 2003

12. Forbes AR, Goldberg ID: Radiation therapy in the treatment of meningioma: the Joint Center for Radiation Therapy experience 1970 to 1982. J Clin Oncol 2:1139-1143, 1984

13. Gill SS, Thomas DG, Warrington AP, Brada M: Relocatable frame for stereotactic external beam radiotherapy. Int J Radiat Oncol Biol Phys 20:599-603, 1991

14. Glaholm J, Bloom HJ, Crow JH: The role of radiotherapy in the management of intracranial meningiomas: the Royal Marsden Hospital experience with 186 patients. Int J Radiat Oncol Biol Phys 18:755-761, 1990

15. Goldsmith BJ, Wara WM, Wilson CB, Larson DA: Postoperative irradiation for subtotally resected meningiomas. A retrospective analysis of 140 patients treated from 1967 to 1990. J Neurosurg 80:195-201, 1994

16. Henzel M, Gross MW, Hamm K, Surber G, Kleinert G, Failing T, et al: Significant tumor volume reduction of meningiomas after stereotactic radiotherapy: results of a prospective multicenter study. Neurosurgery 59:1188-1194, 2006

17. Henzel M, Gross MW, Hamm K, Surber G, Kleinert G, Failing T, et al: Stereotactic radiotherapy of meningiomas: symptomatology, 
acute and late toxicity. Strahlenther Onkol 182:382-388, 2006

18. Jalali R, Loughrey C, Baumert B, Perks J, Warrington AP, Traish $\mathrm{D}$, et al: High precision focused irradiation in the form of fractionated stereotactic conformal radiotherapy (SCRT) for benign meningiomas predominantly in the skull base location. Clin Oncol (R Coll Radiol) 14:103-109, 2002

19. Khoo VS, Adams EJ, Saran F, Bedford JL, Perks JR, Warrington $\mathrm{AP}$, et al: A comparison of clinical target volumes determined by $\mathrm{CT}$ and MRI for the radiotherapy planning of base of skull meningiomas. Int J Radiat Oncol Biol Phys 46:1309-1317, 2000

20. Kollová A, Liscák R, Novotny J Jr, Vladyka V, Simonová G, Janousková: Gamma Knife surgery for benign meningioma. J Neurosurg 10:325-336, 2007

21. Kondziolka D, Flickinger JC, Perez B: Judicious resection and/or radiosurgery for parasagittal meningiomas: outcomes from a multicenter review. Gamma Knife Meningioma Study Group. Neurosurgery 43:405-414, 1998

22. Kooy HM, Dunbar SF, Tarbell NJ, Mannarino E, Ferarro N, Shusterman S, et al: Adaptation and verification of the relocatable Gill-Thomas-Cosman frame in stereotactic radiotherapy. Int $\mathbf{J}$ Radiat Oncol Biol Phys 30:685-691, 1994

23. Kreil W, Luggin J, Fuchs I, Weigl V, Eustacchio S, Papaefthymiou G: Long term experience of gamma knife radiosurgery for benign skull base meningiomas. J Neurol Neurosurg Psychiatry 76:1425-1430, 2005

24. Larson DA, Flickinger JC, Loeffler JS: The radiobiology of radiosurgery. Int J Radiat Oncol Biol Phys 25:557-561, 1993

25. Leber KA, Bergloff J, Pendl G: Dose-response tolerance of the visual pathways and cranial nerves of the cavernous sinus to stereotactic radiosurgery. J Neurosurg 88:43-50, 1998

26. Lee JY, Niranjan A, McInerney J, Kondziolka D, Flickinger JC, Lunsford LD: Stereotactic radiosurgery providing long-term tumor control of cavernous sinus meningiomas. J Neurosurg 97: 65-72, 2002

27. Malik I, Rowe JG, Walton L, Radatz MW, Kemeny AA: The use of stereotactic radiosurgery in the management of meningiomas. Br J Neurosurg 19:13-20, 2005

28. Mendenhall WM, Morris CG, Amdur RJ, Foote KD, Friedman WA: Radiotherapy alone or after subtotal resection for benign skull base meningiomas. Cancer 98:1473-1482, 2003

29. Milker-Zabel S, Zabel A, Schulz-Ertner D, Schlegel W, Wannenmacher M, Debus J: Fractionated stereotactic radiotherapy in patients with benign or atypical intracranial meningioma: longterm experience and prognostic factors. Int J Radiat Oncol Biol Phys 61:809-816, 2005

30. Miralbell R, Linggood RM, de la Monte S, Convery K, Munzenrider JE, Mirimanoff RO: The role of radiotherapy in the treatment of subtotally resected benign meningiomas. J Neurooncol 13:157-164, 1992

31. Mirimanoff RO, Dosoretz DE, Linggood RM, Ojemann RG, Martuza RL: Meningioma: analysis of recurrence and progression following neurosurgical resection. J Neurosurg 62:18-24, 1985

32. Morita A, Coffey RJ, Foote RL, Schiff D, Gorman D: Risk of injury to cranial nerves after gamma knife radiosurgery for skull base meningiomas: experience in 88 patients. J Neurosurg 90: 42-49, 1999

33. Nagele T, Petersen D, Klose U, Grodd W, Opitz H, Voigt K: The "dural tail" adjacent to meningiomas studied by dynamic contrastenhanced MRI: a comparison with histopathology. Neuroradiology 36:303-307, 1994
34. Nicolato A, Foroni R, Alessandrini F, Bricolo A, Gerosa M: Radiosurgical treatment of cavernous sinus meningiomas: experience with 122 treated patients. Neurosurgery 51:1153-1161, 2002

35. Petit JH, Hudes RS, Chen TT, Eisenberg HM, Simard JM, Chin LS: Reduced-dose radiosurgery for vestibular schwannomas. Neurosurgery 49:1299-1307, 2001

36. Pollock BE, Stafford SL, Utter A, Giannini C, Schreiner SA: Stereotactic radiosurgery provides equivalent tumor control to Simpson Grade 1 resection for patients with small- to mediumsize meningiomas. Int J Radiat Oncol Biol Phys 55:1000-1005, 2003

37. Roche PH, Regis J, Dufour H, Fournier HD, Delsanit C, Pellet W, et al: Gamma knife radiosurgery in the management of cavernous sinus meningiomas. J Neurosurg 93 (3 Suppl):68-73, 2000

38. Rogers L, Jensen R, Perry A: Chasing your dural tail: factors predicting local tumor control after gamma knife stereotactic radiosurgery for benign intracranial meningiomas: In regard to DiBiase et al: (Int J Radiat Oncol Biol Phys 2004;60:1515-1519). Int J Radiat Oncol Biol Phys 62:616-619, 2005

39. Schlegel W, Pastyr O, Bortfeld T, Gademann G, Menke M, MaierBorst W: Stereotactically guided fractionated radiotherapy: technical aspects. Radiother Oncol 29:197-204, 1993

40. Selch MT, Ahn E, Laskari A, Lee SP, Agazaryan N, Solberg TD, et al: Stereotactic radiotherapy for treatment of cavernous sinus meningiomas. Int J Radiat Oncol Biol Phys 59:101-111, 2004

41. Shin M, Kurita H, Sasaki T, Kawamoto S, Tago M, Kawahara N, et al: Analysis of treatment outcome after stereotactic radiosurgery for cavernous sinus meningiomas. J Neurosurg 95:435-439, 2001

42. Stafford SL, Perry A, Suman VJ, Meyer FB, Scheithauer BW, Lohse CM, et al: Primarily resected meningiomas: outcome and prognostic factors in 581 Mayo Clinic patients, 1978 through 1988. Mayo Clin Proc 73:936-942, 1998

43. Stafford SL, Pollock BE, Foote RL, Link MJ, Gorman DA, Schomberg PJ, et al: Meningioma radiosurgery: tumor control, outcomes, and complications among 190 consecutive patients. Neurosurgery 49:1029-1038, 2001

44. Taylor BW Jr, Marcus RB Jr, Friedman WA, Ballinger WE Jr, Million RR: The meningioma controversy: postoperative radiation therapy. Int J Radiat Oncol Biol Phys 15:299-304, 1988

45. Tishler RB, Loeffler JS, Lunsford LD, Duma C, Alexander E III, Kooy HM, et al: Tolerance of cranial nerves of the cavernous sinus to radiosurgery. Int J Radiat Oncol Biol Phys 27:215-221, 1993

46. Torres RC, Frighetto L, De Salles AA, Goss B, Medin P, Solbert $\mathrm{T}$, et al: Radiosurgery and stereotactic radiotherapy for intracranial meningiomas. Neurosurg Focus 14(5):E5, 2003

47. Whittle IR, Smith C, Navoo P, Collie D: Meningiomas. Lancet 363:1535-1543, 2004

Manuscript submitted August 6, 2007.

Accepted September 12, 2007.

Address correspondence to: Jay S. Loeffler, M.D., Massachusetts General Hospital, Department of Radiation Oncology, 100 Blossom Street, Cox 3, Boston, Massachusetts 02114. email: jloeffler@ partners.org. 